\title{
ALGUNAS REFLEXIONES ACERCA DE LA DERIVACIÓN Y LA PERSPECTIVA DIACRÓNICA EN SU ESTUDIO
}

\author{
Luis Alexis Amador Rodríguez \\ CEO Rey Juan Carlos
}

\section{Resumen}

La formación de palabras está constituida por una serie de procedimientos morfológicos regulares cuyos resultados interesan al léxico, pero con un carácter gramatical innegable. No obstante, este dominio de las lenguas no se entiende sin atender a aspectos diacrónicos que contribuyen a la comprensión de su configuración actual. La ampliación de las posibilidades expresivas de los diferentes procedimientos derivativos del español, la influencia del préstamo de unidades pertenecientes a otras lenguas y la pervivencia de irregularidades obligan a introducirse en el terreno de la historia para dar cabal explicación a todos estos fenómenos. Palabras clave: derivación, diacronía, formación de palabras, supletivismo.

SOME REFLECTIONS ABOUT THE DERIVATION AND THE DIACHRONIC PERSPECTIVE ON ITS STUDY

\section{Abstract}

The word formation is made up of regular morphological procedures whose results concern the lexikon, but with a clear grammatical nature. However, this linguistic domain remains incomprehensible without a diachronic point of view that contributes to the understanding of its current configuration. The increase of expressive possibilities with the different Spanish derivative procedures, the influence of loanwords and the persistence of irregularities compel us to introduce the history of the Spanish language to explain all these phenomenona. KEYwORDs: derivation, diachrony, word formation, supletivism. 


\section{INTRODUCCIÓN}

Los procedimientos de formación de palabras de nuestra lengua (derivación y composición) están dominados por los principios de la analogía y de la transparencia, de suerte que sirven para la ampliación de parcelas extensas del vocabulario sin tener que recurrir a otros procedimientos neológicos como el préstamo. Esto es lo que ha dado en llamarse gramaticalización del léxico, es decir, la capacidad que tiene la lengua de utilizar procedimientos sistemáticos, análogos a los de la gramática, con la finalidad de acrecentar el número de sus unidades léxicas. Así, por ejemplo, con el sufijo - alse pueden crear series en principio ilimitadas de adjetivos a partir de sustantivos del tipo adverbial, formal, global, otoñal, sensacional, terrenal, etc., en los que prima la expresión de una especial relación entre la base y el derivado a través del morfema derivativo.

No obstante, lo primero que llama la atención al acercarse al dominio de la formación de palabras y, más concretamente, al terreno de la derivación es el hecho de que muchas palabras derivadas muestren una gran irregularidad formal respecto a sus bases (e. g.: recibir > recepción, obispo > episcopal, perdiz > perdiguero, leche > lácteo, etc.). Además, hay una gran cantidad de elementos que pueden catalogarse como afijos en nuestra lengua. Se da el caso, en este punto, de que ni siquiera hay acuerdo entre los estudiosos acerca de su número, baste echar una ojeada a obras de referencia como las de Alemany Bolufer (1920), Bosque y Demonte (1999), Almela (1999), Rainer (1993), Pharies (2002), Morera (2005) o la nueva gramática de la Real Academia Española (2009), en las que la nómina de unidades afijales entre ellas puede variar en centenares, divergencia atribuible a diferencias en los presupuestos teóricos y metodológicos de los que parte cada una. Además, muchos de esos afijos se emplean para el mismo cometido en una suerte de competencia, tal como sucede con los denominados apreciativos o con las series conceptuales de los clasificados como nombres de agente, de instrumento o de lugar. Podría decirse que en este campo reina un lujo expresivo desenfrenado que contraviene el principio de economía más básico y que es producto de la acumulación de siglos en los diferentes estadios históricos del español, en los que la evolución de la propia lengua y el préstamo de materiales tomados de otras lenguas han interactuado para configurar el estado actual. Es por esto por lo que en el dominio de la formación de palabras se hace imprescindible tener en cuenta la diacronía para explicar la sincronía, siguiendo al respecto un criterio ponderado como el que se recoge en la Nueva gramática de la lengua española de la RAE (2009, I: 337 y ss.) o propugnan Clavería (2013) y Pena Seijas (2013). En este breve trabajo nos interesa delinear algunas propuestas de análisis del dominio de la derivación que sirvan para desentrañar la dialéctica existente entre el pasado y el presente a partir de algunas cuestiones que consideramos clave y, de esa forma, ayudar en el tratamiento y estudio de los elementos susceptibles de ser considerados morfemas derivativos. Esbozaremos, por lo tanto, algunos principios de corte teórico y metodológico con los que nos hemos enfrentado al análisis de esta parcela del dominio de las lenguas y que se hacen a modo de reflexiones sobre nuestro propio trabajo. 


\section{GRAMATICALIZACIÓN Y LEXICALIZACIÓN}

Antes de entrar en materia, no obstante, deben tenerse en cuenta dos aspectos definidores del dominio de la formación de palabras en general y de la derivación en particular, que ya hemos adelantado en la introducción, a saber: la gramaticalización del léxico y la lexicalización de la gramática (Coseriu 1981; Lüdtke 1978; Laca 1986). Ambos conceptos nos ayudarán a establecer el marco teórico y metodológico en el que nos movemos para adentrarnos en el estudio de los procedimientos morfológicos de derivación. La gramaticalización del léxico hace referencia a la índole gramatical de los procedimientos que se aplican sobre el léxico para incrementarlo con nuevas unidades. La determinación de las bases léxicas a través de los esquemas de formación de palabras es muy parecida en su naturaleza a la que operan las reglas de la morfosintaxis en el nivel oracional, aunque no llegue a confundirse plenamente con ellas. Es decir, lo que constituye el significado fundamental de un procedimiento derivativo consiste en una aportación semántica que introduce un modo especial o perspectiva específica desde la que entender un referente concreto. Por su parte, la lexicalización se produce cuando la unidad creada entra a formar parte del léxico. Allí las nuevas creaciones reciben, al ser empleadas, una serie de determinaciones que se corresponden con necesidades designativas o de uso que fijan cómo ha de entenderse cada nueva formación, lo que contribuye a diluir los tipos fundamentales y regulares en una casuística heterogénea que hace difícil frecuentemente su tratamiento dentro de una perspectiva más cercana a la gramática y sistematizable.

Así pues, desde nuestro punto de vista, la definición de un procedimiento de formación de palabras se ha de realizar fundamentalmente a través del establecimiento de la relación que mantienen entre sí los elementos que constituyen la nueva unidad (derivada o compuesta). Dicha relación se hace visible mediante la representación semántica que proyecta el procedimiento sobre la noción general de las bases léxicas y se caracteriza por la oposición con respecto a los otros procedimientos morfológicos de la formación de palabras con los que compite en el sistema. En este sentido, seguimos el principio simple enunciado por Benveniste (1948: 6) en su estudio sobre los nombres de agente en indoeuropeo, según el cual, para definir el significado de un procedimiento morfológico de formación de palabras, hay que recurrir a la comparación de los que coinciden en su uso y evitar incluir en la definición rasgos que contradigan cualquiera de sus variantes de contenido. Es decir, por un lado, hay que tener presente que para establecer los valores lingüísticos de los diferentes procedimientos de una lengua habrá que tomar en consideración con qué otros procedimientos comparten el mismo cometido y, por otro lado, habrá que abstenerse de incorporar como elementos definitorios de las unidades de la lengua los que son producto de las necesidades designativas o del uso y que puedan entrar en contradicción con otros usos de la misma unidad. Por ejemplo, un mismo derivado puede designar distintas realidades, como sucede con cargador 'persona que se encarga de conducir cargas' y 'aparato de conexión eléctrica para cargar baterías', pero no sería acertado plantear que haya sido creado a través de un procedimiento distinto para cada designación con la adjunción del sufijo -dorla, pues con ello se lograría úni- 
camente la disgregación del esquema derivativo en función del referente que tome la nueva unidad creada con él. Precisamente el abuso de este principio referencialista en el dominio de la formación de palabras ha llevado a postular la existencia de procedimientos homónimos sin tener en cuenta que lo que define el valor de una unidad lingüística no es el objeto o estado de cosas al que apunta, sino el especial modo de significar de cada una al referirse a la realidad designada (Morera 2005: 5-6). De modo que para entender el significado de los morfemas derivativos habría que ir más allá de la clasificación tradicional en series conceptuales, por ejemplo, del tipo de nombres de agente, nombres de instrumento, nombres abstractos, etc.

Precisamente, el principio que considera primordial el contraste entre los morfemas que coinciden en el uso para perfilar su función o valor de lengua hace necesaria la consideración de los aspectos evolutivos e históricos de la lengua, puesto que la interacción entre los diferentes procedimientos morfológicos para la creación de palabras está sujeta a los avatares del idioma. Ello contribuye a definir la realidad lingüística de los esquemas de derivación en cada uno de los estadios del sistema de la lengua.

\section{MOTIVACIÓN FRENTE A NO MOTIVACIÓN}

En el terreno de los procedimientos de formación de palabras y, particularmente, en el de la derivación, es fundamental el concepto de motivación relativa, tal como aparece recogido en Saussure. La relación entre forma y significado es la que permite reconocer la existencia de un esquema derivativo, es decir, la combinación de una base léxica y de uno o varios afijos, como se aprecia en alunizar, inservible, pregonero, restablecer, etc. A partir del análisis de dichas combinaciones pueden establecerse distintos grados de motivación y/o de regularidad de los procedimientos derivativos, así como entender otros fenómenos relacionados con la necesidad de dotar de motivación a lo que los hablantes interpretan que debe estarlo (Ullmann 1986 [1962]: 115-119). Asimismo, por un lado, nos permite establecer los fenómenos de alomorfia o de variación formal de un determinado procedimiento, asegurados por la expresión de un mismo contenido, o, en el caso de la expresión de contenidos diferentes, la existencia de procedimientos distintos; y, por otro lado, nos ayuda a determinar cuáles son los esquemas derivativos productivos frente a los que no lo son (Rainer 2013).

Así pues, siguiendo la propuesta de Fruyt (1984), podemos establecer tres grandes grupos de derivados en función de su grado de motivación: derivados motivados, derivados semimotivados y palabras no motivadas. Las últimas quedan fuera de nuestro interés, en tanto que no es posible reconocer un esquema o estructura de constituyentes, incluso aunque en algún estadio muy anterior pudiera decirse de ellas que sí lo tuvieron: imbécil, inane, lábaro, etc. Así pues, únicamente atenderemos los dos primeros casos. 


\subsection{Derivados motivados}

Estos son los derivados más regulares desde el punto de vista formal y semántico y, por ende, se corresponden con los esquemas derivativos más productivos. Puede suceder que la base de derivación sea un lexema libre y realizado en el léxico de la lengua y que los afijos pueden aislarse fácilmente respecto a ella. Los fenómenos morfofonológicos en el punto de unión de la base y de los afijos son predecibles y se someten sin dificultad a regla desde una perspectiva sincrónica. Así, por ejemplo, los derivados regulares en -ble se adjuntan mayoritariamente a temas verbales con una vocal temática inflexionada en el punto de unión en los casos de bases verbales pertencientes a la segunda conjugación, e. g.: amable, temible, fundible, etc. Este es el terreno de la analogía y la tendencia es la uniformidad y unificación de los procedimientos que comprende. Es, por tanto, una fuerza determinante en el devenir histórico de las lenguas. No obstante, también puede ocurrir que nos encontremos con los fenómenos de supletivismo y de base ligada, en los que la regularidad de los procedimientos derivativos queda comprometida por cuestiones de norma, de uso o de marcado léxico y, en último término, por razones de corte histórico' ${ }^{1}$.

Empezaremos primero por los casos en los que la analogía desempeńa un papel importante en la evolución diacrónica y funciona desde el interior del propio sistema lingüístico, favoreciendo incluso la ampliación de las posibilidades expresivas de los mecanismos de la lengua, es decir, afectando a la propia variación semántica de los procedimientos derivativos en cuestión. Un ejemplo de ello lo tenemos en la evolución experimentada por el sufijo latino -tor/-ōris en castellano, bajo la forma patrimonial de -dor, que nos servirá para ilustrar varios fenónemos relativos a la actuación de la analogía.

En primer lugar, la analogía favorece naturalmente el establecimiento de nuevos esquemas morfofonológicos, de la manera en que le sucedió de hecho al sufijo - dorla, que se adjunta principalmente a temas verbales con vocal temática sin inflexionar. No obstante, en latín el tema para la formación de derivados en -tor, óris era el propio del supino, lo que explica ejemplos del tipo DUCTOR, ŌRIs (< DUCTUS) 'conductor', SCRIPTOR, ŌRIS (<SCRIP-TUS) 'escritor', SUCESSOR, ŌRIS (< SUCESSUS) 'sucesor', VICTOR, ŌRIS 'vencedor' (< VIC-TUS), etc., formados sobre temas fuertes del supino ${ }^{2}$. La adopción del modelo regular castellano [radical verbal + vocal temática + -dorla] procede de la interpretación de los resultados patrimoniales de los derivados latinos con el sufijo -tor, ōris en nuestra lengua, que, como ha dejado claro Pattison (1975: 111-114), presentan siempre una vocal $(-a-,-e-,-i-)$, por ejemplo: AMATŌREM > amador, BIBǏTŌREM > bebedor, AUDĪTŌREM > oidor ${ }^{3}$. Esto, unido

1 «Si la synchronie permet de décrire les champs de productivité, le reste ne peut être expliqué que par lhistoire» (Molino 1985: 36).

2 Sobre la evolución de la forma latina desde el indoeuropeo puede consultarse para más detalle Watmough (1995-1996).

${ }^{3}$ La absorción de gran parte de los verbos de la tercera conjugación latina por la cuarta (origen de nuestra tercera conjugación) contribuyó a interpretar muchos derivados en -tor de verbos -ěre, 
a la reestructuración de la conjugación verbal en el paso del latín al castellano, hizo que los hablantes rehicieran el modelo regular, tomando las vocales entre la raíz y el sufijo como exponente de la conjugación a la que pertenecía la base. La fuerza de este fenómeno se ha extendido incluso a los derivados de base nominal, adoptando como modelo la derivación a partir de verbos de la primera conjugación, que no en vano es la más productiva en español. Baste citar a modo de ejemplo los casos de caduceador 'rey de armas que publicaba la paz y llevaba en la mano el caduceo' (< caduceo), bacinador 'persona que pide limosna para el culto religioso u obras pías' (< bacin o bacina), bretador 'reclamo o silbo para cazar aves' (< brete), leñador 'persona que corta o vende leña' ( $<$ leña), etc.

Por otro lado, este morfema, inicialmente empleado en la lengua madre para crear sustantivos que designaban de manera exclusiva agentes humanos, pasa a ser utilizado también en la creación de adjetivos de semántica activa a partir de un momento dado, acerca del que no se puede proporcionar una datación exacta ${ }^{4}$, pero que ocurre también en el resto de las lenguas romances además de en español. Naturalmente, es fácil imaginar que la atribución realizada por la categoría sustantiva puede realizarse, a través de la metábasis adjetiva, a cualquier ente nombrado en el seno de un sintagma nominal. El paso estaba en potencia en el propio sistema y bastaba solo con que se diera alguna realización concreta para que el procedimiento derivativo acabara creando, por analogía, también adjetivos. De hecho, en Amador (2009: 146) hemos defendido que los derivados en -dor/a en castellano tienen en la actualidad una función atributiva de carácter primordialmente adjetivo, con posible realización sustantiva. El índice formal de esta función adjetivadora se encuentra en la indeterminación de género del sufijo -dorla, que admite tanto el masculino como el femeninos.

La analogía funciona también, por supuesto, más allá de los cambios de categoría gramatical o la expresión del género, en el desarrollo de la variación semántica de los procedimientos derivativos. Así, continuando con el caso de los derivados en - dor/a en nuestra lengua, las unidades con este sufijo experimentaron una ampliación del campo designativo desde los nombres de agentes humanos administrador, cobrador, sembrador, pescador, etc., a los nombres de instrumento. Este es un fenómeno muy común en diferentes lenguas indoeuropeas y para su explicación se han postulado varias hipótesis. La más difundida tiene que ver con una extensión metafórica que lleva de quien hace algo al objeto que se utiliza para algo. Esto es lo que

como procedentes de verbos en -îre, tal como ocurrió con REPETITOR, ŌRIS (< REPETO, REPETĚre, İTUM) Y FUGITTOR, ŌRIS (<FUGIO, FUGěRE).

${ }^{4}$ Para el latín medieval (Stotz 2000: 273) se ha señalado el empleo atributivo o adjetivo frecuente a partir de las formaciones agentivas femeninas -trix/-icis como sucede en ARMA VICTRICIA 'arma vencedora' o en ULTRICIA BELLA 'guerra vengadora', lo que podría dar una pista sobre el origen de este fenómeno para las lenguas románicas.

${ }^{5}$ La formación del femenino en - a para el sufijo - dorla también es producto de la analogía, puesto que la terminación femenina hipercaracterizada culta -trix/-icis (con su correspondiente patrimonial -driz) ha tenido un uso poco productivo en la historia del castellano. 
encontramos en Meyer-Lübke (1974 [1890-1906]: 611-612) y a partir de él en otros autores. Más recientemente, Rainer (2004), partiendo también de la metáfora, ha propuesto como explicación un mecanismo de aproximación por el que los nombres de instrumento se asemejan a los agentes, lo que pudo servir para la creación directa de aquellos ${ }^{6}$. Asimismo, desde la corriente de la lingüística cognitiva, se ha concebido la existencia de una escala de agentividad que lleva de un iniciador inmediato (agente, fuerza o causa) a uno mediato o instrumental (Dressler 1986; Rifón 1996-1997). Lo cierto es que, desde nuestro punto de vista, de nuevo nos encontramos ante la explotación de una posibilidad contenida en el esquema derivativo a partir de los nombres de agente por su oficio o su profesión que, como bien vio ya Benveniste (1948: 61) en su estudio sobre los nombres de agente en indoeuropeo, se basan en un predicado de futuro o de prospectividad, en un ser para alguna fun-

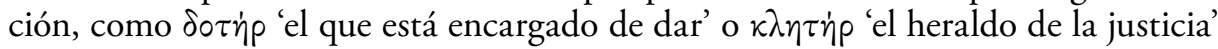
en griego. Desde esa perspectiva hemos formulado la definición semántica para la derivación en -dorla (Amador 2009: 146) como un procedimiento que atribuye a un determinado ser o entidad convertirse en fuente u origen de la realización de un proceso o actividad. Los instrumentos, por tanto, surgen también de este ser para algo, tal como el sabio francés ejemplifica también para el griego y podemos ver en el caso de los derivados espańoles: atizador 'intrumento para atizar el fuego', calentador 'objeto o instrumento para calentar', cargador 'instrumento para cargar', destornillador 'instrumento para destornillar', deponiendo el rasgo [+ humano] presente en el latín clásico, que se convierte en superfluo o en un hecho de habla. Como sucede habitualmente en los callados procesos de transformación de la lengua, es difícil datar a partir de cuándo se produce este hecho, aunque hay designaciones registradas de instrumento en -dorla desde al menos el siglo XIII (Garcés 1986; Morales Ruiz 1998; Pharies 2002; Clavería 2004; Rainer 2004). La asunción de la designación de personas y objetos ha provocado un fenómeno de neutralización que ha ampliado el alcance del valor de lengua del procedimiento derivativo en -dorla desde el latín como lengua madre.

Asimismo, la analogía puede interactuar con el fenómeno del préstamo. Es lo que ha ocurrido con el empleo de -dor/a en español para nombrar lugares. Inicialmente las unidades portadoras del afijo - dor para la designación de espacios o lugares en los que se hace algo eran préstamos procedentes del provenzal o del catalán, lenguas que comparten un formante -dor (pero procedente de -torium) para la creación de nombres de lugar (Diez 1973 [1874]: 328; Garcés 1986: 99; Staib 1988: 142-143), e. g.: corredor, mirador, obrador, parador, etc., o quizás calcos de unidades existentes, principalmente en catalán, como comedor (cat. menjador) o recibidor (cat. rebedor). Su incorporación al castellano permitió la derivación propia en nuestra lengua de nuevos nombres de lugar sin correspondencia en las len-

${ }^{6}$ Rainer (2004) deja en el aire la hipótesis del origen foráneo de los derivados que toman un referente instrumental con -dor, quizás de procedencia catalana o aragonesa, a la manera en que también se ha acreditado para los nombres de lugar. 
guas mencionadas, como abrevador, aselador, colector, distribuidor o pudridor. Sin duda, a este hecho contribuyó el parecido formal con el sufijo productivo autóctono -dor/a (proveniente del latín -tor, óris); de manera que los nombres de lugar en -dor se sienten parte del mismo esquema derivativo y han contribuido a perfilar la función lingüística del procedimiento en la actualidad, aunque la productividad para la designación de lugares se haya frenado por la competencia de otros sufijos con el mismo cometido desde el punto de vista de la norma (principalmente-derola y su variante culta-torio/a). En la explicación de este fenómeno se ha hecho intervenir la metonimia dentro de lo que antes mencionamos como escala de la agentividad, de forma que la designación de lugares con los derivados en -dor/a puede interpretarse como un desplazamiento desde el agente que realiza algo al lugar en el que lo realiza. Por nuestra parte, consideramos que la expresión de la finalidad o la prospectividad permitió la ampliación del espectro designativo del sufijo en cuestión, mediando la incorporación de los préstamos citados, que espolearon la potencialidad dada por el valor de lengua del propio procedimiento morfológico. Baste tener en cuenta que no es extraño que los mismos sufijos que se utilizan para la designación de instrumentos funcionen también para nombrar los lugares que sirven para llevar a cabo una acción concreta, como ocurre con el sufijo -derola en nuestra lengua. Así, por ejemplo, junto a los lugares designados por abrevadero, aparcadero, pudridero, invernadero, etc., tenemos los pertenecientes a la serie de los instrumentos del tipo de atizadero, coladero, majadero, tajadero, etc. En este sentido, consideramos que la coalescencia o el parecido formal entre afijos favoreció el reanálisis por parte de los hablantes y pudo ayudar a ampliar los esquemas de lexicalización de un determinado morfema y a perfilar su valor de lengua.

Dejando a un lado los casos regulares gobernados por la analogía, entre los derivados motivados hemos incluido también los fenómenos de alomorfia y/o supletivismo ${ }^{7}$, que son aquellos en los que la forma del derivado no se puede predecir a partir de la base o lexema autónomo con el que se encuentra relacionado semántica y etimológicamente, como sucede, por ejemplo, con construir > construcción, poseer $>$ posesión, obispo > episcopal, ceniza > cinerario, etc. Los términos derivados mencionados son producto del préstamo, más concretamente, latinismos, que han entrado a formar parte de la familia léxica de sus bases respectivas por esta vía. Pero no mantienen la misma relación que los derivados regulares con el término primario, pues no obedecen a una regla formativa en sincronía. En este sentido, la relación establecida entre la base y el derivado estaría marcada léxicamente, es decir, habría en español una doble articulación del componente de formación de palabras en la que sería necesario distinguir la existencia de una derivación propiamente española y una exclusivamente latina (Coseriu 1981: 53; Clavería 2013). Esta doble arquitectura se extiende a aquellos casos del tipo caballunolequino, en los que hay también

7 Proponemos un tratamiento conjunto de ambos fenómenos en tanto que la diferencia entre la alomorfia y el supletivismo parece más bien una cuestión de grado, que, por supuesto, tiene repercusiones morfológicas, pero de las que no nos vamos a ocupar aquí. 
una unidad patrimonial con la que la serie de derivados cultos mantienen una relación semántico-léxica, pero no etimológica, de manera que hay dos familias léxicas claramente diferenciadas, como se observa en caballo, caballuno, caballero caballería, etc., frente a equino, equitación, ecuestre, etc., que se van entrelazando y alternando.

Al hilo de lo expuesto, sucede frecuentemente que la base de una determinada serie de derivados no se corresponde con una palabra autónoma de la lengua, aunque posea una semántica clara y pueda aislarse en el seno de la familia de palabras a la que pertenece. Es lo que tenemos, por ejemplo, en vulnerar, vulnerario, vulnerable (< lat. vULNUS, ĚRIS); ígneo, ignición (< lat. IGNIS, IS). Ocurre a menudo también en unidades prefijadas, e. g.: conducir, deducir, inducir, reducir, seducir, traducir (sobre el verbo latino DUCo 'llevar, conducir, guiar'). Todos ellos son casos de base ligada o no realizada en nuestra lengua.

Por tanto, cuando tenemos delante ejemplos de supletivismo y de bases ligadas, hay que tomar cada caso como idiosincrásico, aun cuando pueda establecerse algún tipo de generalización como la de que los verbos acabados en /- $\theta \mathrm{ibiR} /$ o /- $\theta$ ebiR/ se nominalizan a partir de un tema acabado en /- $\theta$ ep-/, como sucede en percibir > percepción, recibir > recepción o concebir > concepción. Dicha generalización se abstrae a posteriori como producto de la inducción a partir de la observación de todos los ejemplos similares existentes en nuestra lengua ${ }^{8}$. Sin embargo, no ha dado lugar a un esquema formativo propio del castellano. En este punto es preciso tener en cuenta la distinción entre afijos patrimoniales y afijos cultos o semicultos, de los que nos ocuparemos más adelante cuando abordemos la cuestión de su tratamiento como alomorfos de un mismo morfema o como unidades independientes.

Asimismo, en relación con los aspectos formales referidos a la alomorfia, los supletivismos y a las bases ligadas hemos de introducir algunas reflexiones sobre la interfijación. Con el reconocimiento de la estructura de la palabra a través de la relación significativa que mantienen sus constituyentes, se concluye la existencia de infijos o interfijos, tal como sucede en humareda o polvareda, que se pueden analizar a partir de sus bases respectivas humo y polvo a las que se añade el sufijo -eda con el elemento interfijado -ar-. La asignación de un significado, así como la delimitación de la función de estos elementos, ha interesado a muchos investigadores y el reconocimiento de su estatus ha sido controvertido. Sin embargo, Malkiel (1958: 178 y ss.) ya defendió su necesidad por cuestiones de orden práctico. Según este autor, habría tres posibilidades de tratar los derivados con interfijo: a) aceptando que estamos ante variantes de la raíz o la base, e. g: humar-, polvar- con adjunción de -eda; b) analizando la terminación con el interfijo como sufijo independiente, lo que haría que se tuviera que ampliar la nómina de sufijos, $i$. e.: las raíces humy polv-más-areda; o c) analizando la secuencia en sus diferentes componentes,

${ }^{8}$ Desde la morfología de corte generativista se tienen en cuenta estos fenómenos para escribir las reglas de distribución de los diferentes alomorfos de un morfema (Fábregas 2017). Sin embargo, no ha de olvidarse que este tipo de descripciones parte de lo que Aronoff (1976) denomina marcado léxico, aspecto que puede ponerse en relación con los hechos de norma lingüística, sancionada por el uso y, por tanto, basada en razones de tipo histórico. 
pasando a integrar el primero de los elementos afijados a la base el conjunto de los interfijos, esto es: polv-ar-eda.

La elección de cualquiera de las dos primeras posibilidades de análisis haría más complicada la descripción de la derivación. Parece que hay que dar cabida a la idea de interfijo, ya sea porque es reconocible en la estructura de la palabra como elemento formal sin significado frente a la base y los afijos, ya sea porque es posible asignarle un determinado significado como un morfema más. En este último caso es necesario tener presente que el elemento interfijado forma parte de una cadena derivativa que adjunta morfemas a la base de forma sucesiva. No es, por tanto, un incremento formal sin más, es decir, inmotivado. En dichas unidades es muy usual que los elementos interfijados tengan un carácter apreciativo, eslabones de la cadena derivativa que no se han lexicalizado y que la norma no recoge, como aguacero, vinajera, ventorrero, etc., pudiendo incluso suceder que el morfema apreciativo interfijado rompa la base como en azuquitar (< azúcar). En otros casos, los interfijos se explican por incorporación de préstamos desde otras lenguas, tal como ocurre con cacaotero, cafetero, cocotero, panetero y tetera, procedentes de adaptaciones del francés (Malkiel 1958: 162; Laca 1986: 525; Amador 2009: 238). Unidades que en última instancia han contribuido a crear un modelo para otros derivados que adoptan también el elemento interfijado, e. g.: aguatero, chivitero, pinatero, viñatero, etc. El factor analógico también desempeńa en estos casos de interfijación un papel determinante, puesto que los incrementos formales entre la base y el morfema derivativo pueden ser producto de reanálisis por parte de los hablantes, como se observa en los casos de barrendero, curandero, paseandero, rezandero, etc., creados sobre el modelo de derivados como hacendero (< hacienda), molendero $(<$ molienda) o parrandero (< parranda), lo que ha motivado la aparición del interfijo -nd-.

Ahora bien, es necesario ser prudentes antes de asegurar que un elemento dado es un interfijo. En muchos casos, los que a primera vista podrían ser clasificados como interfijos resultan ser desde una perspectiva histórica producto de derivaciones sucesivas sobre formaciones existentes en estadios anteriores de la lengua, como ocurre en carnicero o panadero, que proceden respectivamente de los sustantivos en desuso carniza y panada y no directamente de carne y pan, como se puede pensar desde una perspectiva sincrónica. De manera que no resultaría adecuado proponer que -iz- y -ad-son elementos formales intercalados entre la base y el sufijo. Otro tanto podría decirse de casos como la serie de términos hornaguero, lombriguera, noguera, peguera y perdiguero, relacionados respectivamente con hornaza, lombriz, nuez, pez y perdiz (madriguera presenta unos cambios formales más acusados frente a matriz). En todos los ejemplos mencionados el derivado en -erola presenta un alomorfo de la base o base ligada, no realizada en español. Como señalan Corominas/Pascual (1980), hornaguero supone la existencia de un no atestiguado *FORNACARIUS, creado sobre el étimo latino FORNAX, ACIS 'horno de cal o de alfarero', del que resulta en espańol hornaza. El término hornaguero es producto, por tanto, de la evolución patrimonial esperable con aspiración y enmudecimiento posterior de la $f$-inicial latina y la sonorización de $/ \mathrm{k} /$ en posición intervocálica. Una explicación similar puede dárseles a lombriguera < lombriz < lat. vulg. LUMBRIX, ICIS < LUMBRICUS, I; noguera < nuez < lat. NUX, NUCIS; peguera < pez<lat. PIX, PICIS y per- 


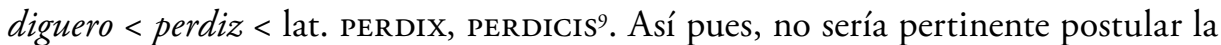
existencia de un interfijo - $g$ - entre el sufijo y la raíz, ya que las diferencias formales se esclarecen recurriendo a la evolución diacrónica. Tampoco sería necesario postular una regla sincrónica que no es operativa en castellano para explicar la transformación de la sibilante final en una velar, pues existen los derivados regulares en - erola hornacero, lombricero y perdicero, aunque con significados diferentes respecto a las unidades de las que venimos hablando, lo que viene a acentuar el marcado léxico de las palabras con la base ligada acabada en consonante velar.

\subsection{Derivados Semimotivados}

Los derivados semimotivados son aquellos en los que parece existir un esquema derivativo, pero no se encuentran realizados en la lengua o bien la base léxica, o bien el afijo, y, por tanto, no se les reconoce una semántica clara.

Así, si nos detenemos en los casos en los que es posible el reconocimiento del afijo, pero no de la base, veremos qué es lo que sucede en las palabras cordero, sarmiento o sazón, en las que es posible aislar sufijos (-erola, -miento y -zón) pertenecientes a nuestra lengua, pero no se puede determinar a qué elementos se adjuntan al no tratarse de unidades léxicas del español y no tener significado. Tratar de identificar una estructura composicional a partir de sus elementos constituyentes en los casos mencionados no tiene sentido alguno y solo la etimología nos da la clave. No se debe confundir, por tanto, con el fenómeno de la base ligada del que nos ocupamos más arriba, pues lo que sucede es que en las unidades de las que estamos tratando las supuestas bases no tienen realidad en la lengua. Así, estas palabras semimotivadas actuarán siempre como término primitivo de una familia léxica, de la manera en que sucede con cordero, corderaje, corderil, corderilla y corderuna; sarmiento, sarmentar, sarmentoso, sarmenticio; y sazón, sazonar.

Para los casos en que es posible reconocer la base de la palabra, pero no el afijo, podemos mencionar lo que ocurre con los derivados latinos en -bulum, $i$ incorporados al castellano, del tipo de conciliábulo, infundíbulo, prostíbulo, vestíbulo, etc. Se trata de préstamos que no han conformado un esquema derivativo productivo en nuestra lengua, aunque podrían servir de modelo para remotivar la terminación latina por parte de los hablantes llegado el caso.

9 En la misma serie entraría el derivado madriguera (< lat. *MATRICARǏAM), relacionado semática y formalmente con el desusado madriz (< lat. MATRIX, ICIs). Su relación con el término matriz se encuentra oscurecida por razones semánticas, de suerte que no puede hablarse de derivación en este caso. 


\section{AFIJOS PATRIMONIALES Y CULTOS: ¿ALOMORFIA O UNIDADES DIFERENTES?}

La estrecha relación a lo largo de su historia entre el español y el latín como lengua madre y lengua de cultura ha motivado que en los estudios de formación de palabras se haya introducido la oposición entre afijos patrimoniales y afijos cultos. La distinción entre unos y otros obedece a una razón eminentemente diacrónica: los denominados afijos patrimoniales son aquellos que han seguido la evolución formal esperada desde el latín al español, mientras que los cultos (o semicultos) se han introducido en etapas posteriores, por lo que no se han sometido (o se han sometido de manera parcial en el caso de los semicultos) a las reglas evolutivas seguidas por los patrimoniales. Esta diferenciación es de gran ayuda, no solo en castellano, sino en todas las lenguas romances, para explicar las peculiaridades morfológicas de las unidades que contienen a unos y a otros; puesto que la presencia de un afijo de tipo culto delata en la mayoría de las ocasiones el carácter de préstamo de las palabras en cuestión.

No obstante, algo que es preciso plantearse cuando nos encontramos ante derivativos patrimoniales y derivativos cultos relacionados desde el punto de vista etimológico es si han de considerarse como alomorfos de un único morfema o como morfemas funcionalmente diferentes. Desde nuestro punto de vista, sobre esta cuestión hay que establecer unos criterios claros para no ampliar de manera innecesaria el inventario de morfemas derivativos en nuestra lengua. Así pues, para la determinación de la existencia o no de alomorfia entre formas patrimoniales y cultas en el terreno de la derivación nos vamos a servir de las siguientes condiciones, que han de operar de forma conjunta:

a) Existe semejanza clara en cuanto al rendimiento funcional o categorial entre formas patrimoniales y cultas. En caso contrario, podríamos estar frente a procedimientos distintos. Es decir, ambas formas afijales sirven o han servido para la formación de palabras pertenecientes a las mismas categorías verbales. Pongamos por caso las unidades léxicas creadas con la terminación sufijal patrimonial-dor/a (alternador, pescador, innovador, retador, etc.) y las formadas con las terminaciones cultas -torla y -sorla (agresor, expositor, invasor, relator, etc.). En estos casos tenemos palabras pertenecientes a la categoría sustantiva con cada una de las terminaciones, así como términos pertenecientes a la categoría de los adjetivos. Puede haber diferencias cuantitativas entre unidades con afijo patrimonial y con afijo culto, tal como ha constatado Laca (1986: 530-532) para los derivados en -erola y en -ariola, ya que se asegura que estos últimos tienden a presentar más frecuentemente la categoría adjetiva. Pero se trata solo de una cuestión de cantidad, no cualitativa o del sistema lingüístico, probablemente condicionada por factores de uso. Así pues, al examinar las formaciones con una y otra forma afijal nos percatamos de que no existen diferencias sustanciales entre los patrones de lexicalización, puesto que hay ejemplos de adjetivos de relación tanto con -erola como con -ariola (financiero, bancario), de adjetivos de comportamiento (callejero, hospitalario), referencia a personas por su actividad (jardinero, notario), beneficiarios 
(heredero, destinatario), nombres de colectivos (refranero, cuestionario), etc. Si quisiéramos establecer una distinción en el nivel del sistema o de la lengua deberíamos acudir al siguiente criterio para fundamentarla.

b) No hay un valor semántico propio y exclusivo de cada uno de ellos correspondiente a una diferencia en el aspecto formal del afijo. Este es un criterio de peso para distinguir los alomorfos de un morfema de aquellos casos en los que nos hallamos frente a morfemas diferentes. Es el caso de -mientol-mento, por un lado, y -menta, por otro. Todas estas terminaciones están relacionadas etimológicamente: -mento es la forma culta de -miento, como demuestra la ausencia de diptongación, y -menta en su origen era la forma del plural de los sustantivos neutros latinos en -mentum, $i$, hoy día una terminación femenina. Si se comparan las formaciones existentes en nuestra lengua con unas y otras terminaciones, se observará el contraste entre el modo de significar de las formas masculinas y de la femenina. Esta última comporta un valor de intensificación o colectivización, tal como se ve en cornamenta, faldamenta, impedimenta, osamenta, etc. Todas estas formas comportan un matiz de 'colectividad' o 'intensificación', y resalta especialmente cuando se les opone un doblete con la terminación -mento del tipo de impedimento 'obstáculo, embarazo o estorbo para algo' / impedimenta 'bagaje que suele llevar la tropa e impide la celeridad de las marchas y las operaciones'. Por tanto, habrá que considerar la terminación -menta como un morfema derivativo distinto de -miento/-mento, entendiéndose en nuestra opinión como la forma marcada de la oposición mencionada.

\section{CONCLUSIÓN}

La inclusión de la perspectiva diacrónica en los estudios sobre formación de palabras es, desde nuestro punto de vista, una necesidad insoslayable para entender la sincronía de cualquier lengua. Si no se tiene en cuenta la aportación de la evolución histórica, se corre el riesgo de interpretar los hechos de un estadio concreto de forma ciega y con poco sentido. Naturalmente, no se trata de favorecer un etimologicismo a todo trance, pues hay que saber conjugar la evolución lingüística con el sentir de los hablantes, atendiendo en todo caso adecuadamente a los fenómenos de motivación que tienen su fundamento en lo que podemos concebir como el deseo de hacer claro lo que se ha vuelto opaco. Así pues, es preciso contar siempre con todos los elementos de juicio que nos permitan dar una explicación cabal de la sincronía. Por supuesto, se hace necesario realizar estudios más pormenorizados de los diferentes procedimientos de formación de palabras y, en el terreno de la derivación, como ya apuntara Pharies (2002: 16), se hace indispensable llevar a cabo estudios que contemplen la competencia existente entre diferentes morfemas para explicar su función lingüística y su comportamiento en cuanto mecanismos de creación de nuevas palabras. 


\section{BIBLIOGRAFÍA}

Alemany Bolufer, José (1920): Tratado de la formación de palabras en castellano, Madrid: Suárez. Almela Pérez, Ramón (1999): Procedimientos de formación de palabras en español, Barcelona: Ariel.

Amador Rodríguez, Luis Alexis (2009): La derivación nominal en español: nombres de agente, instrumento, lugar y acción, Frankfurt am Main: Peter Lang.

Aronoff, Mark (1976): Word Formation in Generative Grammar, Cambridge: The MIT Press.

Bosque, Ignacio y Violeta Demonte, coord. (1999): Gramática descriptiva de la lengua española, 3, Madrid: Espasa Calpe.

Clavería Nadal, Gloria (2004): «Los caracteres de la lengua en el siglo xirI: el léxico», en Rafael Cano (coord.), Historia de la lengua española, Madrid: Barcelona, 473-496.

Clavería Nadal, Gloria (2013): «La formación de palabras y el cultismo», en Isabel Pujol Payet (ed.), Formación de palabras y diacronía, A Coruña: Universidade da Coruña, Anexos de Revista de Lexicografia 19, 49-68.

Corominas, Joan y José Antonio Pascual (1980): Diccionario Crítico Etimológico Castellano e Hispánico, Madrid: Gredos.

Coseriu, Eugenio (1981): Principios de semántica estructural, Madrid: Gredos.

DiEz, Friedrich (1973 [1874]): Grammaire des langues romanes, II, Genève/Marseille: Slatkine/Laffitte.

Dressler, Wolfgang (1986): «Explanation in natural morphology, illustrated with comparative and agent-noun formation", Linguistics 24: 519-547.

FÁbregas, Antonio (2017): La morfología. El análisis de la palabra compleja, Madrid: Síntesis.

Garcés, María del Pilar (1986): «Aportación al estudio de los préstamos galorromances en las Partidas de Alfonso X El Sabio", Epos. Revista de Filología 2, UNED: 89-101.

LACA, Brenda (1986): Die Wortbildung als Grammatik des Wortschatzes. Untersuchungen zur spanischen Subjektnominalisierung, Tübingen: Gunter Narr.

LACA, Brenda (1993): «Las nominalizaciones orientadas y los derivados españoles en -dor y -nte», en Soledad Varela Ortega (ed.), La formación de palabras, Madrid: Taurus, 180-204.

LüdtKe, Jens (1978): Prädikative Nominalisierungen mit Suffixen mi Französischen, Katalanischen und Spanischen, Tübingen: Max Niemeyer.

LüDtKe (2004): Romanische Wortbildung, Tübingen: Stauffenberg.

Malkiel, Yakov (1958): «Los interfijos hispánicos», en Estructuralismo e Historia. Miscélanea homenaje a André Martinet, II, Universidad de La Laguna, 107-199.

Malkiel, Yakov (1988): «Las peripecias españolas del sufijo latino -oriu, -oria», Revista de Filología Española LXVIII: 217-255.

Meyer-Lüвкe, Wilhelm (1974 [1890-1906]): Grammaire des langues romanes, II, Genève / Marseille: Slatkine / Laffitte.

Morales Ruiz, María del Carmen (1998): «La evolución de los sufijos -dor y -dero: un caso de amalgama morfológica para la expresión del género", Estudi General. Revista de la Facultat de Lletres de la Universitat de Girona 17: 145-171.

Morera, Marcial (2005): La complementación morfológica en español, Frankfurt am Main: Peter Lang. 
Morreale, Margherita (1963-1964): «El sufijo -ero en el Libro de Buen Amor», Archivo de Filologia Aragonesa XIV-XV: 235-244.

Nieto Ballester, Emilio (2013): Introducción a la etimología, Madrid: Síntesis.

PAscual, José Antonio y Nieves SÁnchez (1992): «Una forma particular de amalgama morfológica: Notas sobre la historia de -dor y -dero en español», en Homenaje a Eugenio de Bustos, Universidad de Salamanca, 675-698.

Pena Seijas, Jesús (2013): «Sobre formación de palabras y familias léxicas: relaciones entre sincronía y diacronía», en Isabel Pujol Payet (ed.), Formación de palabras y diacronía, A Coruña: Universidade da Coruña, Anexos de Revista de Lexicografía 19, 91-108.

Pharies, David (2002): Diccionario etimológico de los sufijos españoles y otros elementos finales, Madrid: Gredos.

RaIner, Franz (1993): Spanische Wortbildungslehre, Tübingen: Max Niemeyer.

Rainer, Franz (2004): «Del nombre de agente al nombre de instrumento y de lugar en espańol: ¿cuándo y cómo?», Iberorromania 59, 97-122.

RAINER, Franz (2013): «Formación de palabras y analogía: aspectos diacrónicos», en Isabel Pujol Payet (ed.), Formación de palabras y diacronía, A Coruña: Universidade da Coruña, Anexos de Revista de Lexicografía 19, 141-169.

Real Academia Española (2009): Nueva gramática de la lengua española. Morfología y sintaxis, Madrid: Espasa.

Real Academia Española (2014): Diccionario de la lengua española, Madrid: Espasa.

Sтаів, Peter (1988): Generische Komposita. Funktionelle Untersuchungen zum Französischen und Spanischen, Tübingen: Max Niemeyer.

STOTz, Peter (2000): Handbuch zur lateinischen Sprache des Mittelalters, II, München: C.H. Beck'sche.

Ullmann, Stephen (1986 [1962]): Semántica. Introducción a la ciencia del significado, Madrid: Aguilar.

Watmough, Margaret T. (1995-1996): «The suffix -tor-: agent-noun formation in Latin and the other Italic languages», Glotta 73: 80-115. 
